\title{
A heuristic approach to the weakly interacting Bose gas
}

\author{
Thomas Nattermann \\ Institut für Theoretische Physik, Universität zu Köln, Zülpicher Str. 77, 50937 Köln, Germany*
}

(Dated: July 6, 2021)

\begin{abstract}
Some thermodynamic properties of weakly interacting Bose systems are derived from dimensional and heuristic arguments and thermodynamic relations, without resorting to statistical mechanics.
\end{abstract}

PACS numbers:

\section{INTRODUCTION.}

In a previous article ${ }^{1}$ I have derived the thermodynamic properties of ideal quantum gases solely from dimensional arguments, the Pauli principle and thermodynamic relations, without resorting to statistical mechanics. In the present publication I will extend this approach to weakly interacting bosons.

Starting point is the Hamiltonian of interacting bosons in $d$ dimensions

$$
\mathcal{H}=\int d^{d} x \Psi^{\dagger}\left(-\frac{\hbar^{2}}{2 m} \nabla^{2}+\frac{1}{2} v_{0} \Psi^{\dagger} \Psi\right) \Psi
$$

Here $\Psi^{\dagger}(\mathbf{x})$ and $\Psi(\mathbf{x})$ are the creation and annihilation operators of the Bose field in $d$-dimensions. $m$ denotes the mass of the Bose particles. $v_{0}>0$ is the strength of the interaction potential $V(\mathbf{x})=v_{0} \delta(\mathbf{x})$ of the bosons. Main reason for choosing here a strictly local interaction is to keep the number of parameters small, which only makes a dimensional analysis possible. However, for low energy phenomena this approximation is not unreasonable.

It is convenient to express $v_{0}$ in terms of a scattering length $b$ which follows from dimensional analysis:

$$
b=\left(\frac{m v_{0}}{S_{d} \hbar^{2}}\right)^{1 /(d-2)}, \quad d>2 .
$$

$S_{d}$ is a prefactor with $S_{3}=4 \pi$. In $d \leq 2$, where (2) diverges with $v_{0} \rightarrow 0$, the scattering length behaves as $b \sim q^{-1}$ where $q$ denotes the transferred momentum since there is always a bound state ${ }^{2}$. However, throughout this paper we will stay in $d>2$ dimensions.

For $v_{0} \equiv 0$ the state of the system is completely described by the occupation numbers $n_{\mathbf{p}}$ of the single particle states which are characterized by their momentum $\mathbf{p}$ and energy $\epsilon_{0}(\mathbf{p})=\mathbf{p}^{2} /(2 m)$. Since the particles of the ideal Bose gas do not occupy any volume and since there is no Pauli principle for bosons, in the ground state all particles can have the same position. One conclusion one can draw from this is that the pressure cannot depend on the volume ${ }^{1}$. There are no collective excitations. This situation is completely changed in the presence of

\footnotetext{
*e-mail: natter@thp.uni-koeln.de
}

even a very weak repulsive interaction. The particles have now a finite cross-section and occupy a finite volume. Local density fluctuations will propagate because of the repulsive forces between bosons. This behavior results in the occurrence of a branch of sound waves i.e. acoustic phonons. The existence of acoustic phonons in the weakly interacting system will be the main assumption of the present considerations.

In the hydrodynamic limit, i.e. for frequencies $\omega \ll$ $\tau_{\text {relax }}^{-1}$ where $\tau_{\text {relax }}$ is a typical relaxation time of the interacting gas, the existence of sound waves follows from the particle number conservation and Euler's equation (Newton's equation for liquids) in a linear approximation ${ }^{3}$. In addition we have to assume here that these sound waves persist in the collision-less limit $\omega \gg \tau_{\text {relax }}^{-1}$, i.e. go over into acoustic phonons. For completeness I remark that besides of ordinary (first) sound in superfluids there is second sound which propagates at $T=0$ with the velocity $c_{2}=c / \sqrt{3}$. Second sound is a hydrodynamic mode which does not exist in the collision-less limit $\stackrel{4}{\text {. }}$

\section{II. $\mathbf{T}=\mathbf{0}$}

We begin with the properties of the ground state. In the non-interacting case all particle are in the state of lowest energy $\epsilon_{0}=0$ and $\left\langle\Psi^{\dagger} \Psi\right\rangle=n_{0}=n$ where $n=$ $N / V$. Here $N$ and $V$ denote the total number of particles and the volume, respectively. Pressure and the chemical potential vanish in the ground state.

Phonons. As discussed already I will now assume that the excitations above the ground state can still be characterized by the momentum $\mathbf{p}$ (an assumption which parallels Landaus picture of a Fermi fluid $\left.{ }^{4}\right)$ but that the dispersion relation $\epsilon(\mathbf{p})$ is changed with respect to $\epsilon_{0}(\mathbf{p})$ such that

$$
\begin{aligned}
\epsilon(\mathbf{p}) \approx c|\mathbf{p}|, & p \lesssim p_{0} \\
\epsilon(\mathbf{p}) \approx \frac{\mathbf{p}^{2}}{2 m}, & p>p_{0} .
\end{aligned}
$$

The sound velocity $c$ and the cross-over momentum $p_{0}$ have to be determined self-consistently. Clearly at $p=p_{0}$

$$
c p_{0} \approx \frac{p_{0}^{2}}{2 m}, \text { i.e. } p_{0} \approx 2 m c .
$$

I ignore here and in the following all factors of order unity since their determination is beyond the accuracy of our 
considerations. It is also evident that for vanishing interaction the linear branch (3) of the dispersion relation should disappear. As long as the energy $p^{2} /(2 m)$ of the free bosons is much larger than $v_{0} n$ (compare (1)) the effect of the interaction is weak and the free boson dispersion relation (4) will essentially remain unchanged. In the opposite case a linear dispersion relation (3) is expected. It is therefore obvious that a second relation for the determination of $p_{0}$ is given by 17

$$
\frac{p_{0}^{2}}{2 m} \approx v_{0} n \approx m c^{2}
$$

which gives with (5)

$$
c=\mathcal{C}_{c}(d) \sqrt{\frac{v_{0} n}{m}} \sim \frac{\hbar}{m \xi_{0}}, \quad p_{0} \sim \sqrt{v_{0} n m} \sim \frac{\hbar}{\xi_{0}} .
$$

The relation (7) with $\mathcal{C}_{c}(3)=1$ agrees for $p \ll p_{0}$ with the well-known result $5,6,7$. Clearly the constant $\mathcal{C}_{c}(d)$ (as well as other constants to come) remains undetermined in the present approach. Apparently $\xi_{0} \approx h / p_{0}$ plays the role of a fundamental length scale of the interacting system besides of the mean boson distance $a=n^{-1 / d} . \xi_{0}$ is the smallest length scale on which collective behavior is still seen. In the superfluid phase it corresponds to the bare correlation or healing length. The assumption of weak interaction (which will be used everywhere below) can be written as

$$
\frac{a}{\xi_{0}}=\left(\frac{b}{a}\right)^{(d-2) / 2} \ll 1, \quad a=n^{-1 / d} .
$$

Zero point fluctuations. The degrees of freedom which are involved in the collective phonon excitations have a non-vanishing zero point energy since they correspond to an ensemble of harmonic oscillators. Hence they cannot contribute to the condensate. The depletion of the number of particles $N_{0}=N n_{0}$ in the condensate is therefore given by

$$
n_{E>0}=n-n_{0}=\sum_{p<p_{0}} 1=\mathcal{C}_{n}(d) n\left(\frac{b}{a}\right)^{\frac{d(d-2)}{2}},
$$

which agrees with the exact result in $d=3$ dimensions if we choose $\mathcal{C}_{N}(d=3)=\frac{8}{3 \sqrt{\pi}} \approx 1.5 \stackrel{8}{\text {. }} . N_{0}$ denotes the number of particles in the condensate. Thus, contrary to the non-interacting case, the Heisenberg uncertainty relation in conjunction with the repulsive interaction enforces that a finite fraction of the system is in excited states.

The energy of the zero point fluctuations of the phonons follows analogously from:

$$
\sum_{p<p_{0}} \frac{1}{2} \hbar c|\mathbf{p}| \sim N_{E>0} c p_{0}
$$

The total ground state energy $E_{0}$ consists then of the interaction of the particles in the condensate and the zero point fluctuations of the phonons

$$
\begin{aligned}
E_{0} & =\frac{1}{2} v_{0} N_{0} n_{0}+\frac{1}{2} \sum_{p<p_{0}} \hbar c|\mathbf{p}|= \\
& =\frac{v_{0} N_{0}^{2}}{2 V_{0}}\left[1+\mathcal{C}_{E}(d)\left(\frac{b}{a}\right)^{d(d-2) / 2}\right] .
\end{aligned}
$$

With $\mathcal{C}_{E}(3)=\frac{128}{15 \sqrt{\pi}} \approx 4.8$ the last relation agrees with the exact result 6 . From (11) follows the chemical potential 10 .

$$
\mu=\frac{\partial E_{0}}{\partial N}=v_{0} n_{0}\left[1+\frac{d+2}{4} \mathcal{C}_{E}(d)\left(\frac{b}{a}\right)^{d(d-2) / 2}\right]
$$

The compressibility $\kappa=\partial n / \partial \mu$ is then

$$
\kappa^{-1}=v_{0}\left[1+\frac{d(d+2)}{8} \mathcal{C}_{E}\left(\frac{b}{a}\right)^{d(d-2) / 2}\right]
$$

Finally we calculate the pressure

$$
p=-\frac{\partial E_{0}}{\partial V} \approx \frac{1}{2} v_{0} n_{0}^{2} \sim \frac{\hbar^{2}}{m a^{d+2}}\left(\frac{b}{a}\right)^{d-2}
$$

It is instructive to express the results (9) - (14) in terms of the healing length (8).

\section{III. $\mathbf{T}>\mathbf{0}$}

Ideal gas. We begin again with a a brief review of the situation in the absence of any interaction ${ }^{1}$. Then at temperatures below the Bose condensation temperature of the ideal gas,

$$
T_{c, 0}=\mathcal{C}_{T_{c}} \frac{\hbar^{2}}{m a^{2}}
$$

where $\mathcal{C}_{T_{c}}=3,31^{11}$, of the order $N\left(a / \lambda_{T, 2}\right)^{d}$ particles are outside of the condensate. Here $\lambda_{T, 2}=\hbar / \sqrt{m T}$ denotes the thermal de Broglie wave length of the non-relativistic Bose particles. The pressure follows as $p \sim T / \lambda_{T, 2}^{d}$ etc., $\operatorname{see}^{\frac{1}{1}}$.

Weak interaction, low T. For non-zero interaction

$$
v_{0} n_{0} \approx m c^{2} \approx \frac{\hbar^{2}}{m \xi^{2}} \sim T_{c, 0}\left(\frac{b}{a}\right)^{d-2} \ll T_{c, 0}
$$

sets a second energy scale. As long as $T \ll m c^{2}$ the relevant part of the excitation spectrum is essentially linear with a thermal de Broglie wave lengthํㅜㄹ

$$
\lambda_{T, 1}=\frac{\hbar c}{T} \sim \frac{T_{c, 0}}{T} \frac{a^{2}}{\xi_{0}} \sim a \frac{T_{c, 0}}{T}\left(\frac{b}{a}\right)^{(d-2) / 2} .
$$

Since there is no phonon number conservation, $\lambda_{T, 1}$ has the meaning of the average spacing between the phonons. 
Since phonons behave as non-interacting relativistic particle we obtain their entropy $S$ by using the relation 1 $S=\mathcal{C}_{S}(d) V / \lambda_{T, 1}^{d}$ for relativistic particles of energy $T$. The exact expression is $\mathcal{C}_{S}(3)=4 \pi^{2} / 90 \approx 0.44 \underline{12}$. Thus their energy $E$ is given by $E_{0}=\frac{d}{d+1} \mathcal{C}_{S}(d) V \frac{T}{\lambda_{T, 1}^{d}}$ and hence we get for the total free energy in the range $T \ll m c^{2} \sim T_{c, 0}(b / a)^{d-2} \underline{14}$

$$
E=\frac{n_{0}^{2} v_{0} V}{2}\left[1+\left(\frac{b}{a}\right)^{\frac{d(d-2)}{2}}\left[\mathcal{C}_{E}+\mathcal{C}_{S}\left(\frac{T}{m c^{2}}\right)^{d+1}\right]\right]
$$

in agreement with the so-called Popov approximation ${ }^{6.15}$. The finite temperature corrections are small, as has to be expected. Specific heat, internal energy, pressure etc. follow from that.

Superfluid density. At zero temperature the whole Bose liquid is superfluid. At finite $T$ however, part of it has normal viscous properties. To obtain the result for the normal and superfluid density, $n_{n}$ and $n_{s}$, respectively, we follow the ideas of Landau and Khalatnikov 16 : We decompose the particle current density in a normal and a superfluid one

$$
\mathbf{j}=n_{s} \mathbf{v}_{s}+n_{n} \mathbf{v}_{n}, \quad n_{s}+n_{n}=n .
$$

Here $\mathbf{v}_{s}$ and $\mathbf{v}_{n}$ denote the velocity of the normal and the superfluid component, respectively, in the laboratory frame. In the frame in which the superfluid is at rest the current is

$$
\mathbf{j}_{0}=\mathbf{j}-\mathbf{v}_{s} n=n_{n}\left(\mathbf{v}_{n}-\mathbf{v}_{s}\right) .
$$

$m \mathbf{j}_{0}$ denotes the mass current density (i.e. the momentum density) of all particles which do not belong to the superfluid. This is the net momentum density resulting from all phonon degrees of freedom. It can be written as

$$
n_{n}\left(\mathbf{v}_{n}-\mathbf{v}_{s}\right)=\hbar^{-d} \int d^{d} p \frac{\mathbf{p}}{m} n_{p h}\left(c p+\mathbf{p}\left(\mathbf{v}_{s}-\mathbf{v}_{n}\right)\right)
$$

where $n_{p h}$ denote the phonon distribution function (which we don't have to know!). We expand the r.h.s. up to linear terms in $\delta \mathbf{v}=\mathbf{v}_{s}-\mathbf{v}_{n}$, multiply the whole equation by $\delta \mathbf{v}$ and perform the angular average. This gives

$$
\begin{aligned}
n_{n} & =\frac{1}{m d} \int d^{d} p p^{2} \frac{\partial n_{p h}}{\partial c p}=\frac{d+1}{d} \frac{1}{m c^{2}} \int d^{d} p c p n_{p h}(p) \\
& =n \mathcal{C}_{S}(d)\left(\frac{T}{m c^{2}}\right)^{d+1}\left(\frac{b}{a}\right)^{\frac{d(d-2)}{2}}=n \frac{S}{N} \frac{T}{m c^{2}} .
\end{aligned}
$$

In the last steps we integrated by parts and expressed the resulting integral in terms of the energy and entropy, respectively. It is remarkable that the last relation is independent of dimension. Clearly the superfluid component has zero entropy. .

\section{Acknowledgment}

It is a pleasure to acknowledge helpful advice on the preparation of this paper by Jens O. Andersen, Sergey Artemenko, Andreas Engel, Axel Pelster, Aleksandra Petkovits, Zoran Ristivojevic, Friedmar Schütze and Matthias Vojta.
1 T. Nattermann, "A scaling approach to ideal quantum gases" Am. J. Phys 73, 349 (2005).

${ }^{2}$ In three dimensions (2) agrees with the textbook result in the limit of small wave vectors. For scattering in two dimensions see

D.S. Petrov and G.V. Shlyapnikov, "Interatomic collisions in tightly confined Bos gas", Phys. Rev. A 64, 12706 (2001).

3 L.D. Landau and E.M. Lifshitz, "Fluid Dynamics", 2nd edition, Pergamon Press Oxford, $\S 64$ (1987).

4 P.W. Anderson, "Basic Notions of Condensed Matter Physics", Benjamin/Cummings Publishing Company, Menlo Park, California (1984).

${ }^{5}$ Most of the results for the weakly interacting Bose gas were originally obtained by T.D. Lee, K. Huang and C.N. Yang in "Eigenvalues and Eigenfunctions of a Bose System of Hard Spheres and Its Low-Temperature Properties", Phys. Rev. 106, 1135-46 (1957).

${ }^{6}$ We refer here to the widespread textbook of L.D. Landau and E.M. Lifshitz, "Statistical Physics, Part 2", second edition, Pergamon Press Oxford (1981), eq. (25.11).

7 More results on the weakly interacting Bose gas can be found in J.O. Andersen, "The Weakly Interacting Bose Gas", Rev. Mod. Phys. 76, 599-639 (2004).

8 Reference ${ }^{6}$, eq. (25.19).

9 Reference ${ }^{6}$, eq. (25.14).

10 Reference ${ }^{6}$, eq. (25.15).

11 L.D. Landau and E.M. Lifshitz, "Statistical Physics, Part 1", third edition, Pergamon Press Oxford (1980), eq.(62.2).

12 Reference $^{11}$, eq. (63.13).

13 Reference ${ }^{6}$, eq. (27.10).

14 Reference ${ }^{6}$, eq. (22.4).

15 V.N. Popov, "Functional Integrals in Quantum Field Theory and Statistical Physics", Reidel. Dortrecht (1983)

16 see in particular I.M. Khalatnikov "An Introduction to the Theory of Superfluidity", W.A. Benjamin Inc, New York, Amsterdam (1965).

17 Some of the formulas resemble relativistic counterparts, e.g. $\xi$ plays the role of the Compton wave length, $m c^{2}$ that of the rest energy etc., but the crossover from nonrelativistic to relativistic behavior is opposite to the conventional one: particles with low momenta behave as relativistic, those with large momenta as non-relativistic ones. 